\title{
CAMBIOS ESTRUCTURALES DEL ÁNGULO DE LA CÁMARA ANTERIOR EN EL GLAUCOMA CONGÉNITO. COMPARACIÓN CON EL DESARROLLO NORMAL
}

\section{STRUCTURAL CHANGES OF THE ANTERIOR CHAMBER ANGLE IN PRIMARY CONGENITAL GLAUCOMA WITH RESPECT TO NORMAL DEVELOPMENT}

\author{
ROJAS B ${ }^{1}$, RAMÍREZ AI ${ }^{2}$, DE-HOZ R ${ }^{1}$, SALAZAR JJ ${ }^{2}$, RAMÍREZ JM ${ }^{1}$, TRIVIÑO A ${ }^{1}$
}

\begin{abstract}
RESUMEN
Objetivo: Comparar los hallazgos morfológicos observados en ángulos normales con los observados en ángulos de pacientes con glaucoma congénito primario, para intentar clarificar los mecanismos patogénicos de esta enfermedad, así como explicar el éxito del tratamiento quirúrgico en alguno de estos pacientes.

Métodos: Se utilizaron ojos adultos normales procedentes de cadáveres y fragmentos quirúrgicos procedentes de trabeculectomías, de pacientes con glaucoma congénito primario que habían sido tratados previamente con una goniotomía. Los tejidos fueron procesados para su examen a microscopía óptica (MO) y microscopía electrónica de barrido (MEB).

Resultados: En los glaucomas congénitos encontramos: 1) inserción alta del iris; 2) No se observa una membrana pre-trabecular, sino que comparando con los ángulos normales las trabéculas aparecen ensanchadas con disminución de los espacios inter-
\end{abstract}

\begin{abstract}
Purpose: To compare findings of normal angles with those from primary congenital glaucoma in order to clarify the pathogenic mechanisms of the disease and to explain the success of surgical treatment in some of these patients.

Methods: Adult normal eyes from cadavers and fragments of surgical trabeculectomies from patients with primary congenital glaucoma previously treated with goniotomy were studied. Eyes were processed for examination using light microscopy and scanning electron microscopy techniques. Results: The following was evident in congenital glaucoma: 1) high iris insertion; 2) no observable pretrabecular membrane, but enlarged trabeculae with diminished inter-trabecular spaces; 3) Schlemm's canal (SC) apparently normal, with vacuoles in the wall indicating normal functioning; 4) sectioning of the abnormal trabecular tissue during goniotomy allowed repositioning of the angle structures and hence the appearance of the angular recess.
\end{abstract}

\footnotetext{
Recibido: 14/7/05. Aceptado: 20/2/06

Instituto de Investigaciones Oftalmológicas Ramón Castroviejo. Universidad Complutense. Madrid. España.

1 Doctor en Medicina.

2 Doctor en CC. Biológicas.

Proyecto subvencionado por Red Temática de Investigación Cooperativa CO3/13 del Instituto de Salud Carlos III.

Correspondencia:

Alberto Triviño Casado

Instituto de Investigaciones Oftalmológicas Ramón Castroviejo

Facultad de Medicina, Pab. VI, 4 . $^{\text {a }}$ planta

Universidad Complutense de Madrid

28040 Madrid

España

E-mail: atrivino@med.ucm.es
} 
trabeculares; 3) el canal de Schlemm (CS) es aparentemente normal, con vacuolas en su pared que indican un funcionamiento normal del mismo; 4) la sección del tejido trabecular anormal que produce la goniotomía, permite el reposicionamiento de las estructuras del ángulo y por tanto la aparición del receso angular.

Conclusiones: En el glaucoma congénito primario las anomalías de las estructuras trabeculares no siempre se acompañan de un desarrollo anómalo del CS y de los canales colectores. Este hecho podría explicar que la goniotomía sea un tratamiento con éxito en estos glaucomas.

Palabras clave: Ojo, canal de Schlemm, malla trabecular, microscopía electrónica, glaucoma congénito.
Conclusions: Anomalies of the trabecular structures in primary congenital glaucoma do not always parallel an abnormal development of the SC and the collector channels. This fact could explain the success of goniotomy in this type of glaucoma (Arch Soc Esp Oftalmol 2006; 81: 65-72).

Key words: Eye, Schlemm's canal, trabecular meshwork, congenital glaucoma, electron microscopy.

\section{INTRODUCCIÓN}

El glaucoma congénito es una enfermedad pediátrica caracterizada por un incremento en la presión intraocular (PIO), secundario a un desarrollo anómalo de las estructuras del ángulo de la cámara anterior. El aumento de PIO produce alteraciones morfológicas que pueden conducir a la ceguera. Se han propuesto diferentes clasificaciones (1) y se han postulado varias hipótesis, que intentan explicar la fisiopatología del glaucoma congénito primario. Barkan (2) y Worst (3) describieron la presencia de una membrana, que cubría el ángulo y se extendía desde la línea de Schwalbe (LS) hasta la malla trabecular bloqueando la salida del humor acuoso. Allen et al (4) postularon que el ángulo de la cámara anterior no se abría completamente, debido a que se producía un clivaje incompleto y una pobre diferenciación y organización de las células del área trabecular. Shaffer (5) defendía la existencia de una inserción anómala del iris en el área más permeable de la malla trabecular, debido a una separación incomplete del tejido durante el desarrollo. Maumenee (6) observó una inserción anormal de los haces longitudinales y circulares del músculo ciliar en las fibras trabeculares, que tendían a comprimir el espolón escleral y que por tanto, comprimían el canal de Schlemm (CS). Otros autores han descrito la presencia de masas de material amorfo o fibroso, en la porción endotelial de la malla trabecular y en los espacios inter-trabeculares de la malla trabecular corneo-escleral $(7,8)$. Por último, se ha postulado el desarrollo incompleto o la ausencia del CS $(5,9,10)$ en algunos casos. Esta falta de consenso en la etiopatogenia del glaucoma congénito, podría explicar porqué los glaucomas congénitos presentan comportamientos diferentes y distintas respuestas ante los tratamientos.

El propósito de este trabajo es estudiar, con técnicas histológicas y microscopía electrónica de barrido (MEB), el ángulo de la cámara anterior de ojos con glaucoma congénito y compararlo con el de ojos humanos normales, para intentar clarificar los mecanismos patogénicos de esta enfermedad y tratar de explicar el éxito del tratamiento quirúrgico que se produce en algunos de estos pacientes.

\section{SUJETOS, MATERIAL Y MÉTODOS}

Para la realización de este estudio, se utilizaron 12 ojos de adultos normales, procedentes de donaciones para trasplante de córnea y 4 fragmentos de piezas de trabeculectomías de pacientes con glaucoma congénito primario, que habían sido tratados previamente con una goniotomía. Los tejidos fueron procesados para su estudio con microscopía óptica (10 ojos humanos y dos piezas de trabeculectomía) y microscopía electrónica de barrido (dos ojos humanos y dos piezas de trabeculectomía). De las piezas de trabeculectomía utilizadas: 3 procedían de trabeculectomías realizadas en zonas del ángulo 
no tratadas previamente con goniotomía (2 para su estudio a MO y 1 para MEB), y una, de una trabeculectomía realizada en la zona de transición entre el tejido seccionado por el goniotomo y el tejido sin seccionar (para su estudio a MEB).

Los tejidos fueron procesados utilizándose los siguientes protocolos.

\section{Microscopía Óptica (MO)}

Los ojos fueron fijados en paraformaldehido (PF)

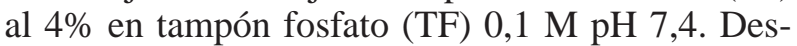
pués de $5 \mathrm{~h}$ de fijación, se separó el segmento anterior del posterior y se mantuvo en la solución fijadora durante $24 \mathrm{~h}$. Posteriormente, el segmento anterior se lavó en tampón fosfato salino (TFS) y se deshidrató en alcoholes de gradación creciente. A continuación se introdujo en tolueno y se incluyó en parafina. Se realizaron secciones sagitales de 6 micras, tiñéndose posteriormente con hematoxilinaeosina, Ünna-Tanzer o azul de tolouidina.

\section{Microscopía electrónica de Barrido (MEB)}

Dos fragmentos de trabeculectomías y dos ojos normales fueron fijados en glutaraldehido $2,5 \%$ en TF $0,1 \mathrm{M}$ ph 7,4 durante $12 \mathrm{~h}$. Posteriormente se lavaron en TF 0,1 M pH 7,4 y fueron postfijados en tetróxido de osmio al $1 \%$ durante $2 \mathrm{~h}$. Después se lavaron en agua destilada, se deshidrataron en acetonas de gradación creciente, se realizó el punto crítico con dióxido de carbono líquido y se metalizaron con oro. A continuación se montaron sobre soportes metálicos, con el ángulo de la cámara anterior mirando hacia arriba. Las muestras fueron observadas y fotografiadas con un microscopio electrónico de barrido.

\section{RESULTADOS}

\section{Adulto normal}

\section{A) $M O$}

En el ángulo iridocorneal del adulto normal, se observó que los espacios intertrabeculares de la malla trabecular córneo-escleral presentaban una morfología elongada. La luz del canal de Schlemm
(CS) era perfectamente visible, así como los colectores externos y los plexos intraescleral y superficial profundo, cuya densidad se distribuía al azar. Los procesos ciliares estaban claramente diferenciados y contenían abundantes vasos sanguíneos.

\section{B) $M E B$}

La malla trabecular se observaba como un tejido triangular que rodeaba a la cámara anterior (fig. $1 \mathrm{~A})$. Su vértice estaba localizado en la membrana de Descemet formando la línea de Schwalbe. La malla trabecular se extendía desde la línea de Sch-

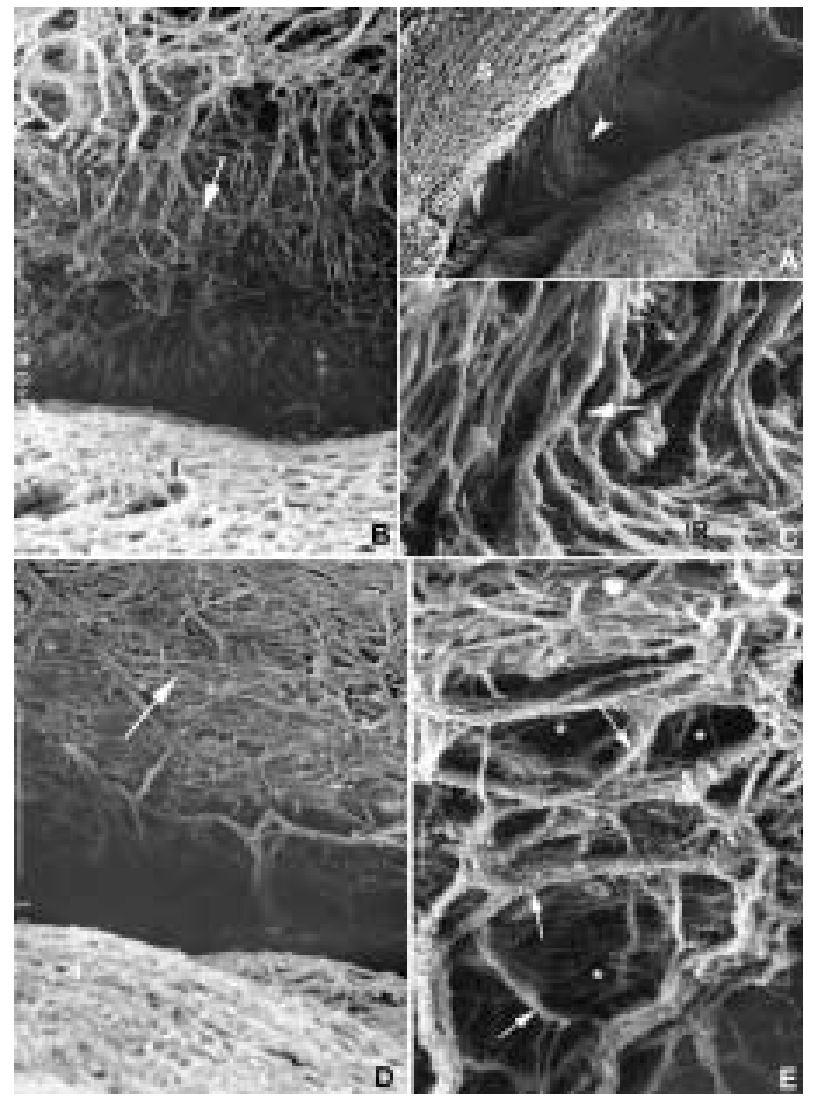

Fig. 1: Microscopía electrónica de transmisión del ángulo irido-corneal del adulto.

A,B: La malla trabecular (cabeza de flecha blanca) se observa como un tejido triangular (A) formado por lamelas pequeñas (flecha) que forman una malla de filtración (B). C: Trabeculum uveal (flecha); D: Trabeculum córneo-escleral (flecha). E: Trabéculas constituidas por bandas aplanadas de tejido (flecha) con aperturas de tamaño variable (asterisco). Iris (I); Esclera (S); Raíz. del iris (IR). 
walbe al estroma del iris y a la superficie anterior del cuerpo ciliar. La malla trabecular estaba formada por pequeñas lamelas, dispuestas unas sobre otras, para formar una rejilla de filtración (fig. 1A y B). En la malla trabecular se identificaron dos porciones, el trabeculum uveal y el trabeculum córneoescleral. El trabeculum uveal estaba localizado próximo a la cámara anterior y sus trabéculas derivaban de la raíz del iris y del cuerpo ciliar (fig. 1C). El trabéculum córneo-escleral consistía en una red de lamelas, que se extendían desde el espolón escleral a la línea de Schwalbe (fig. 1D). Los trabeculum córneo-escleral y uveal externo estaban constituidos por bandas aplanadas de tejido. Entre estas bandas había aberturas angulares de tamaño variable con las esquinas redondeadas (fig. 1E). Estas aberturas estaban orientadas circunferencialmente, paralelas a la superficie del limbo córneo-escleral (fig. 1E). En la capa más interna del trabéculum uveal, próxima a la cámara anterior, las trabéculas presentaban una especie de cordones redondeados que se orientaban radialmente. Estos cordones delimitaban grandes espacios ovales, circulares o romboidales formando una malla (fig. 1C).

\section{Glaucoma congénito}

\section{A) $M O$}

Los fragmentos de las trabeculectomías presentaban masas de material amorfo a nivel de la malla trabecular y en el tejido yuxtacanalicular. Este material, constituido por un material de desecho homogéneo, producía un engrosamiento de las trabéculas y un estrechamiento considerable de los espacios intertrabeculares. El CS no mostraba alteraciones.

\section{B) $M E B$}

En las preparaciones de glaucoma congénito se observaron alteraciones con respecto a la inserción del iris (fig. 2A y B), que se encontraba muy por delante del espolón escleral. En las secciones transversales se observó que la raíz del iris podía insertarse paralela al CS (fig. 2B), o anterior y paralela al CS (fig. 2A), próxima al tejido corneal. Esta inserción anormal determinaba la ausencia de receso angular (figs. 2A-C).
Las trabéculas en forma de cordones del trabeculum uveal y las aplanadas del córneo-escleral, ambas observadas en los individuos normales, no se encontraron en los individuos con glaucoma congénito. En lugar de esto, había un tejido compacto y bastante homogéneo con pequeñas aberturas, que se extendía desde la inserción anómala del iris a la LS (fig. 2C). Este tejido no era diferente al del adulto normal, sino el resultado de una modificación del tejido de la malla trabecular debido al engrosamiento de las trabéculas, que determinaba un estrechamiento de los espacios intertrabeculares (fig. 2C,D).

La pared interna del CS presentaba células endoteliales muy grumosas, lo que podría indicar la presencia de vacuolas gigantes en su interior (fig. 3A).

En una de las piezas de trabeculectomía pudimos analizar la incisión de la goniotomía justo en el límite entre el área tratada y no tratada. El examen de la zona revelaba que el goniotomo había roto el tejido trabecular, permitiendo que el iris rotase hacia atrás. El reposicionamiento del iris abría el ángulo en este área, descubriendo el receso angular (figs. 3B y C).

\section{DISCUSIÓN}

Al igual que otros autores (11-15), nuestro análisis de los fragmentos de las trabeculectomías de los pacientes con glaucoma congénito primario, tratados previamente con goniotomía, ha demostrado cambios estructurales tanto en las trabéculas corneo-esclerales como en las uveales. Estos cambios van a producir una disposición alterada del iris, dando la impresión de una inserción alta del mismo. Decimos que «da la impresión», ya que la inserción del iris tiene una localización normal y lo que determina la apariencia de una inserción alta es la unión de unas trabéculas uveales grandes, por encima o por debajo en el espolón escleral. Esta observación ya había sido descrita por Kupfer and Ross (1971) (15), quienes postularon que residuos de tejido mesodérmico del iris serían los responsables de que la parte del iris cercana al receso angular se adhiera al espolón escleral, dando así la impresión de una inserción alta del iris.

Uno de los aspectos más controvertidos en el glaucoma congénito, es si este tejido constituye una membrana propiamente dicha (2,16-19), o es simplemente el resultado de una estructuración diferen- 

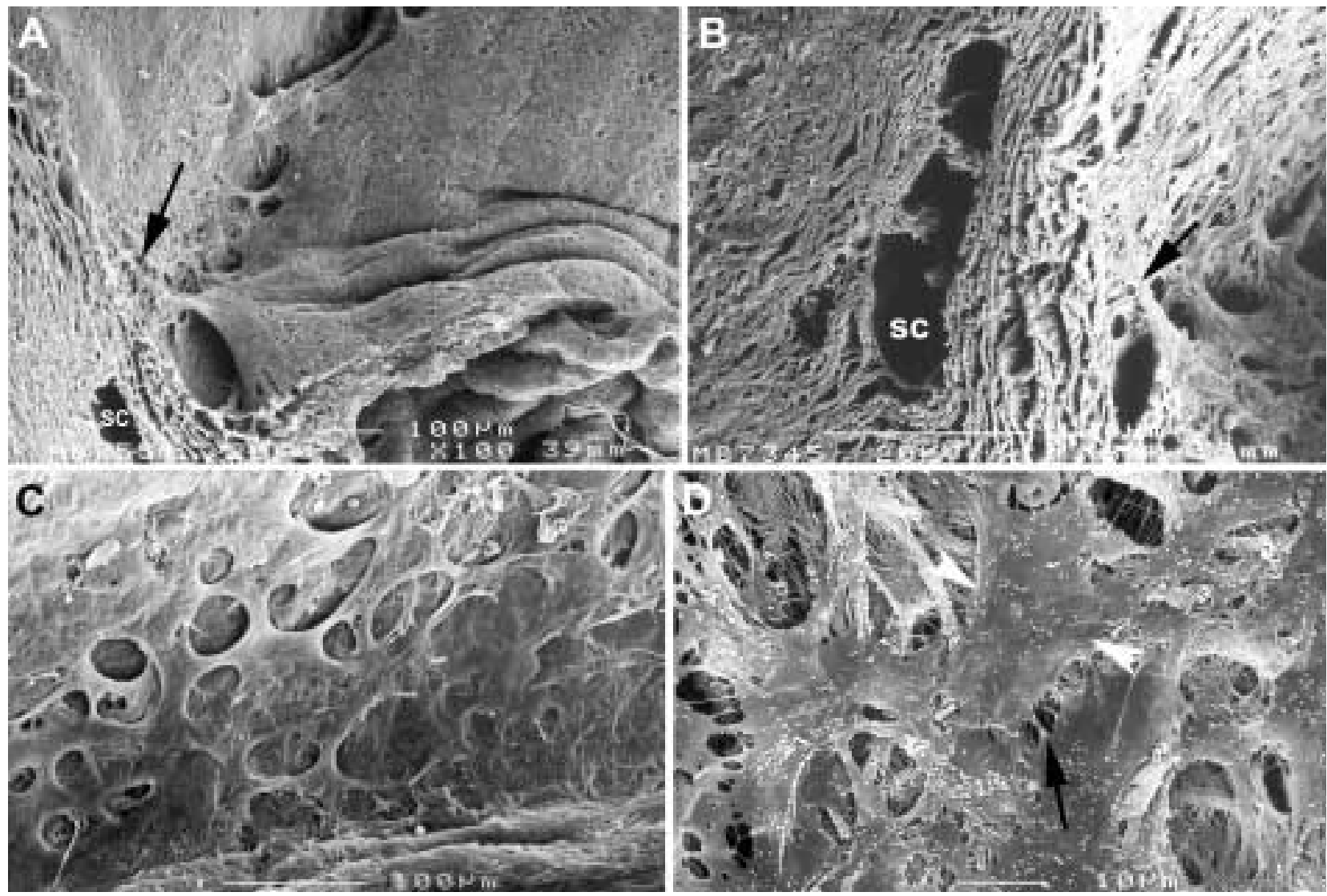

Fig. 2: Microscopía electrónica de transmisión del ángulo irido-corneal del un glaucoma congénito.

A,B: Anomalías en la inserción del iris. A: la raíz del iris se inserta anterior y paralelo (A, flecha) o paralelo (B, flecha) al canal de Schlemm (CS). C,D: Trabéculas engrosadas (cabeza de flecha blancas) con espacios intertrabeculares disminuidos (flecha).

te del tejido trabecular. Coincidiendo con otros autores $(3,15,20,21)$, en nuestras preparaciones no se ha observado una membrana, sino unas trabéculas de mayor tamaño que en los adultos normales. Este tejido procedía de zonas del ángulo que no estaban seccionadas por el goniotomo, ya que las trabeculectomías se hicieron en áreas donde no se había realizado una goniotomía previa. Este aumento en el tamaño de las trabéculas, es debido fundamentalmente a la presencia de un tejido mesenquimal que al reducir los espacios intertrabeculares, favorece el acúmulo de distintos materiales (incluso eritrocitos). Al igual que otros autores (15), se observó este material amorfo tanto en la malla trabecular como en la porción yuxtacanalicular.

Algunos grupos han postulado que en la patogenia del glaucoma congénito se produciría una falta en el desarrollo del CS $(5,9,10)$. En los fragmentos procedentes de las trabeculectomías, no se encon- traron anomalías a MEB en el desarrollo del CS. Muy al contrario, la configuración del CS en los tejidos estudiados era normal, hasta el punto que pudimos observar vacuolas gigantes en la pared interna del mismo.

En uno de los pacientes con glaucoma congénito primario, al que se le había practicado previamente una goniotomía, se seleccionó para la trabeculectomía la zona de transición entre el tejido seccionado por el goniotomo y el tejido sin seccionar. El análisis ultrasestructural de la zona seccionada por el goniotomo demostró, que la configuración de las estructuras trabeculares por detrás del corte eran normales. De acuerdo con Broughton (19), pensamos que el efecto mecánico del corte podría permitir el reposicionamiento de las estructuras angulares. La sección de las trabéculas uveales (las más engrosadas) por el goniotomo, permitiría una caída hacia atrás del iris, dejando ver el espolón escleral 


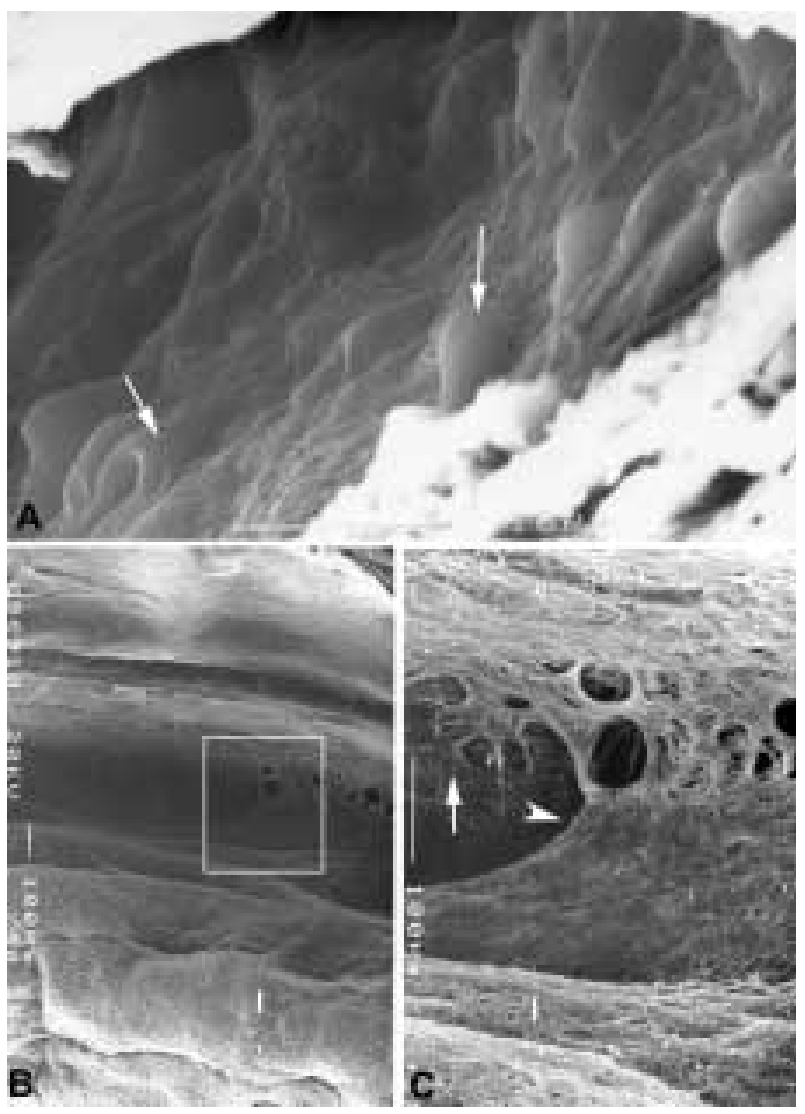

Fig. 3: Microscopía electrónica de transmisión del ángulo irido-corneal de un glaucoma congénito.

A: Células endoteliales prominentes en la pared interna del canal de Schlemm (flecha). B,C: Incisión de una goniotomía en el límite entre la zona tratada y no tratada (recuadro). C: La ruptura del tejido trabecular (cabeza de flecha) permite la rotación del iris hacia atrás, descubriendo el receso angular (flecha). Iris (I).

y la banda ciliar, exponiendo por tanto, el receso angular (20,22). Estas observaciones explicarían el éxito de la goniotomía como tratamiento del glaucoma congénito primario. En nuestra opinión, como la configuración del ángulo es aparentemente normal en estos pacientes, la goniotomía seccionaría el tejido trabecular uveal anormal, permitiendo la evacuación del acuoso sin resistencia. Por el contrario, en aquellos casos de glaucoma congénito primario que no se resuelven con la goniotomía, podría ocurrir que el fallo funcional resida en un aumento de resistencia en las vías post-trabeculares. Este aumento de resistencia podría ser secundario, a un acúmulo de material amorfo en el tejido yuxtacana- licular $(3,14)$, a un desarrollo inadecuado del CS o sus conductos colectores $(5,10)$.

\section{AGRADECIMIENTOS}

Agustín Fernández, del Centro de Microscopía Electrónica «Luis Bru» (Universidad Complutense de Madrid).

\section{BIBLIOGRAFÍA}

1. Hoskins HD Jr, Shaffer RN, Hetherington J. Anatomical classification of the developmental glaucomas. Arch Ophthalmol 1984; 102: 1331-1336.

2. Barkan O. Pathogenesis of congenital glaucoma: gonioscopic and anatomic observation of the angle of the anterior chamber in the normal eye and in congenital glaucoma. Ophthalmology 1955; 40: 1-11.

3. Worst JG. Congenital glaucoma. Remarks on the aspect of chamber angle, ontogenic and pathogenic background, and mode of action of goniotomy. Invest Ophthalmol 1968; 7: 127-134.

4. Allen L, Burian HM, Braley AE. A new concept of the development of the anterior chamber angle; it's relationship to developmental glaucoma and other structural anomalies. AMA Arch Ophthalmol 1955; 53: 783-798.

5. Shaffer RN. Pathogenesis of congenital glaucoma; gonioscopic and microscopic anatomy. Trans Am Acad Ophthalmol Otolaryngol 1955; 59: 297-308.

6. Maumenee AE. The pathogenesis of congenital glaucoma; a new theory. Am J Ophthalmol 1959; 47: 827-859.

7. Hara K. Basic and clinical studies on glaucoma in childhood. Part 5. Fine structure of the trabecular meshwork in congenital glaucoma. Nippon Ganka Gakkai Zasshi 1977; 81: 995-1005.

8. Maul E, Strozzi L, Muñoz C, Reyes C. The outflow pathway in congenital glaucoma. Am J Ophthalmol 1980; 89: 667-673.

9. Tawara A, Inomata H. Developmental immaturity of the trabecular meshwork in congenital glaucoma. Am J Ophthalmol 1981; 92: 508-525.

10. Crombie AL, Cullen JF. Hereditary glaucoma occurrence in five generations of an Edimburgh family. Br J Ophthalmol 1964; 48: 143-147.

11. Anderson DR. The development of the trabecular meshwork and its abnormality in primary infantile glaucoma. Trans Am Ophthalmol Soc 1981; 79: 458-485.

12. Katai N, Urakawa Y, Sato Y, Miyanaga K, Segawa K, Yoshimura N. CHARGE association with congenital glaucoma due to maldevelopment of the anterior chamber angle. Acta Ophthalmol Scand 1997; 75: 322-324.

13. Lin J, Sun W, Li E, Wang S, Bai J, Yang H. Ultrastructural observations of the anterior chamber angle tissues in congenital glaucoma. Yan Ke Xue Bao 1994; 10: 50-56.

14. Sampaolesi R. Anatomía patológica en el glaucoma congénito puro. In: Sampaolesi R. Glaucoma. Buenos Aires. Panamericana; 1991; 771-783. 
15. Kupfer C, Ross $K$. The development of outflow facility in human eyes. Invest Ophthalmol 1971; 10: 513-517.

16. Hansson HA, Jerndal T. Scanning electron microscopic studies on the development of the iridocorneal angle in human eyes. Invest Ophthalmol 1971; 10: 252-265.

17. Lalive d'Epinay S. Congenital glaucoma. Clinical, therapeutic and histological aspects. Adv Ophthalmol 1980; 41: 1-89.

18. Manschot WA. Juxtapapillary retinal angiomatosis--histology of congenital glaucoma-transverse ischaemic optic nerve necrosis in neuroblastoma. Ophthalmologica 1970; 160: 326-329.
19. Broughton WL, Fine BS, Zimmerman LE. Congenital glaucoma associated with a chromosomal defect. A histologic study. Arch Ophthalmol 1981; 99: 481-486.

20. Spencer WH. Glaucoma. In: Spencer WH. Ophthalmic Pathology. An atlas and text book. Philadelphia: WB Saunders Company; 1985; 480-547.

21. Reme C, d'Epinay SL. Periods of development of the normal human chamber angle. Doc Ophthalmol 1981; 51 . 241-268.

22. Gorin G. Developmental glaucoma. A concept based on correlation of gonioscopic findings with clinical manifestations. Am J Ophthalmol 1964; 58: 572-580. 